\title{
INTRODUCCIÓN A PHYSICS AND PHILOSOPHY DE WERNER HEISENBERG
}

\section{INTRODUCTION TO PHYSICS AND PHILOSOPHY OF WERNER HEISENBERG}

Paul Charles William Davies: Universidad Estatal de Arizona. Instituto BEYOND paulcw.davies@gmail.com

\section{CURRÍCULUM VITAE}

Físico, escritor y locutor británico reconocido a escala internacional. Actualmente ocupa la posición de profesor en la Universidad Estatal de Arizona, donde dirige el instituto BEYOND: Center for Fundamental Concepts in Science. Anteriormente ha ocupado cargos académicos en la Universidad de Cambridge, Universidad de Londres, Universidad de Newcastle, Universidad de Adelaida y en la Universidad de Macquarie, Sídney. Sus investigaciones se centran en el campo de la cosmología, teoría cuántica de campos, y astrobiología. Davies considera que un viaje de sólo ida a Marte es una opción viable.

\section{RESUMEN}

La edad dorada de la física ha supuesto un cambio radical en la concepción que los físicos tienen del mundo. Se formularon, como consecuencia, dos teorías novedosas, una del espacio, el tiempo y el movimiento, llamada relatividad, y otra de la naturaleza de la materia y las fuerzas que actúan sobre ella, que desembocó en la teoría de la mecánica cuántica y su principal motor, el principio de incertidumbre, 
que recibe su nombre en honor a Heisenberg, uno de los principales arquitectos de la teoría. La teoría de la relatividad incluye dos teorías (la de la relatividad especial y la de la relatividad general) formuladas por Albert Einstein a principios del siglo XX, que pretendían resolver la incompatibilidad existente entre la mecánica newtoniana y el electromagnetismo. La mecánica cuántica es una de las ramas principales de la Física y uno de los más grandes avances del siglo XX en el conocimiento humano. u aplicación ha hecho posible el descubrimiento y desarrollo de muchas tecnologías, como por ejemplo los transistores.

\title{
PALABRAS CLAVE
}

Física - Teoría de la relatividad - Mecánica Cuántica

\begin{abstract}
The golden age of physics has been a radical change in perception that physicists have of the world. Were made as a result, two novel theories, one of space, time and movement, called relativity, and over the nature of matter and the forces acting on it, which led to the theory of quantum mechanics and main engine, the uncertainty principle, which is named in honor of Heisenberg, one of the main architects of the theory. The theory of relativity includes two theories (special relativity and general relativity) made by Albert Einstein in the early twentieth century, which sought to resolve the incompatibility between Newtonian mechanics and electromagnetism. Quantum mechanics is one of the main branches of physics and one of the greatest twentieth-century advances in human knowledge. or application has enabled the discovery and development of many technologies, such as transistors.
\end{abstract}




\section{KEY WORDS}

Physics - Theory of Relativity - Quantum Mechanics

\section{TEXTO}

En ciencia, las auténticas revoluciones suponen algo más que espectaculares descubrimientos y rápidos avances en nuestra comprensión de las cosas. También logran cambiar los conceptos en los que se basa la disciplina de que se trate. Ese fue el tipo de transformación fundamental que la física experimentó durante los primeros treinta años de este siglo, culminando en lo que se ha venido en llamar la edad dorada de la física. Como consecuencia de ello, la concepción que del mundo tienen los físicos se ha visto radical e irreversiblemente alterada.

Los desarrollos que desencadenaron esta monumental convulsión entrañaban la formulación de dos teorías dramáticamente novedosas. La primera era una teoría del espacio, el tiempo y el movimiento, llamada relatividad. La segunda era una teoría de la naturaleza de la materia y de las fuerzas que actúan sobre ella. Esta última tuvo su origen en la observación de Max Planck de que la radiación electromagnética se emite en paquetes discretos, o cuantos. A partir de esta "teoría cuántica" se elaboró, en los años veinte, una mecánica cuántica general. El autor de este libro desempeñó un papel señero en la temprana formulación de la mecánica cuántica y en la posterior clarificación de sus revolucionarias implicaciones. Aquellos lectores que sepan algo sobre mecánica cuántica sabrán que el famoso "principio de incertidumbre", pieza fundamental de la física cuántica, recibe su nombre en honor a Heisenberg. 
Aunque recientemente se ha escrito mucho sobre los extraños fundamentos conceptuales de la mecánica cuántica, ha de darse especial importancia a estas consideraciones de uno de los principales arquitectos de la teoría. Hasta su misma muerte en 1976, Heisenberg mantuvo sumo interés por la naturaleza del universo cuántico y por las profundas implicaciones filosóficas que de él se derivan. La exposición que sigue es un estudio exhaustivo de estas ideas, junto con una valoración de la teoría de la relatividad y de algunos aspectos de la física nuclear y de partículas. Es un modelo de claridad y una de las explicaciones más lúcidas de la llamada interpretación de Copenague de la mecánica cuántica que se ha convertido en punto de vista generalmente admitido.

El tema central de la exposición de Heisenberg, que se basa en sus clases magistrales Gifford de 1955-56, en la Universidad de San Andrés, en Escocia, es que las palabras y los conceptos normales de la vida diaria pueden perder su significado en el mundo de la relatividad y de la física cuántica. De modo que cuestiones sobre el espacio y el tiempo, o sobre cualidades de los objetos materiales, tales como sus posiciones, que parecen del todo razonables en nuestro lenguaje habitual, no siempre se pueden contestar con pleno sentido. Lo cual tiene, a su vez, profundas implicaciones para la naturaleza de la realidad y para nuestra visión general del mundo.

En muchos aspectos, la convulsión conceptual que exige la teoría de la relatividad puede acomodarse mejor que la debida a la mecánica cuántica. Cierto es que la relatividad contiene algunas ideas extrañas, tales como la dilatación del tiempo y la contracción de la longitud, el espacio curvo y los agujeros negros. También afirma esta teoría que ciertos tipos de preguntas, que suenan perfectamente razonables y plenas de sentido, no se pueden responder sin ambigüedades. Preguntar, por ejemplo, a qué hora ocurre un suceso, o si dos sucesos separados en el espacio ocurren en el mismo momento, puede que no se pueda responder tal cual se plantea, puesto que la teoría nos dice que no existe un tiempo universal absoluto, ni un 
concepto universal de simultaneidad. Tales cosas son relativas y han por tanto de estar referidas a un marco de referencia específico antes de que la pregunta pueda tener sentido. Pero aunque estas ideas sean extrañas y nada familiares, no nos resulta obvio que sean absurdas. Ni presentan auténticos problemas de interpretación. Por esta razón, la teoría de la relatividad, tanto en su versión especial como general, ha de considerarse incontrovertible.

Probablemente el problema filosófico más profundo que presenta la teoría de la relatividad es la posibilidad de que el universo pueda haber tenido su origen en un momento finito del pasado y que este origen represente el nacimiento, no sólo de la materia y la energía, sino también del espacio y del tiempo. En efecto, la lección fundamental de la teoría de la relatividad es que el espacio y el tiempo no son meramente la arena en la que se representa el drama del universo, sino parte del reparto. Es decir, el espacio-tiempo es tan parte del universo físico como la materia; de hecho, la materia y el espacio-tiempo se hallan íntimamente entrelazados. Como Heisenberg comenta, la idea de que el tiempo no se estire hacia atrás por toda la eternidad sino que fuese creado con el universo fue anticipada en el siglo V por San Agustín. Existe por tanto un correlato científico de la creación ex nihilo de la tradición cristiana. Pero la violencia que se hace a nuestro concepto de causalidad física es profunda, y es sólo en fechas muy recientes, dentro del contexto de la cosmología cuántica moderna (desarrollada tras la muerte de Heisenberg) cuando se ha presentado un cuadro satisfactorio del origen del espacio-tiempo.

A diferencia de lo que sucede con la teoría de la relatividad, la mecánica cuántica nos presenta problemas filosóficos y conceptuales de mucho mayor calado, y es de estos problemas de los que Heisenberg se ocupa aquí con tanta claridad. Debería insistirse desde el principio en que la mayoría de los estudiantes aprenden mecánica cuántica por prescripción docente y la aplican sin tener que verse jamás envueltos en embrollo filosófico alguno. La aplicación práctica de la mecánica cuántica conlleva éxitos 
extraordinarios y ha penetrado muchas áreas de la ciencia y la tecnología modernas. Nadie cuestiona lo que la teoría predice, únicamente lo que significa.

En el corazón de la revolución cuántica está el principio de incertidumbre de Heisenberg. Nos dice, más o menos, que todas las cantidades físicas que se pueden observar están sujetas a fluctuaciones impredecibles, de modo que sus valores no están precisamente definidos. Considérese, por ejemplo, la posición x y el momento $\mathrm{p}$ (N. de T.: producto de la masa por la velocidad) de una partícula cuántica tal como un electrón. El experimentador es libre de medir cualquiera de estas cantidades con arbitraria precisión, pero no es posible que obtenga valores precisos de las dos simultáneamente (N.de T.: siempre que la medida simultánea se haga en la misma dirección del espacio. Es posible medir con precisión valores simultáneos de la posición en una dirección del espacio y el momento -velocidad- en cualquier otra dirección perpendicular a la anterior). La extensión, o incertidumbre, de sus valores, denotada por Dx y Dp respectivamente, es tal, que su producto DxDp no puede ser inferior a un cierto número constante. De manera que mayor precisión en la posición exige renunciar a mayor precisión en el momento, y viceversa. La constante que así surge (llamada constante de Planck en honor a Max Planck) es numéricamente muy pequeña, así que los efectos cuánticos generalmente sólo son importantes en el dominio atómico. En la vida cotidiana no los percibimos.

Es esencial darse cuenta de que esta incertidumbre es inherente a la naturaleza y no meramente el resultado de limitaciones tecnológicas en la medida. No es que el experimentador sea sencillamente demasiado torpe para medir simultáneamente posición y momento. La partícula simplemente no posee simultáneamente valores precisos de estos dos atributos. Estamos acostumbrados a la incertidumbre en muchos procesos físicos - por ejemplo, en el mercado de valores o en termodinámicapero en estos casos la incertidumbre se debe a falta de información más que a alguna limitación fundamental en cuanto a lo que podemos saber sobre estos sistemas. 
La incertidumbre tiene profundas implicaciones. Por ejemplo, significa que una partícula cuántica no recorre un camino bien definido en el espacio. Un electrón puede abandonar un lugar A y llegar hasta un lugar B, pero no es posible atribuirle una trayectoria concreta que una los dos puntos. De modo que el popular modelo del átomo, con los electrones dando vueltas alrededor del núcleo en distintas órbitas, es ciertamente falaz. Heisenberg nos dice que tal modelo puede valer para que nos formemos una cierta imagen mental, pero es una imagen que sólo guarda un vago parecido con la realidad.

Qué posición y momento queden así difuminados conduce a un indeterminismo inherente al comportamiento de los sistemas cuánticos. Hasta la más completa información sobre un sistema (que puede ser tan simple como una sola partícula moviéndose en libertad) es generalmente insuficiente para que sea posible una predicción precisa sobre el comportamiento del sistema. Así que puede que dos sistemas inicialmente idénticos evolucionen de manera dispar. Por ejemplo, el experimentador puede disparar un electrón hacia un objetivo y encontrarse con que se difunde hacia la izquierda, y entonces, al repetir el experimento bajo exactamente las mismas condiciones, encontrarse con que el siguiente electrón se difunde hacia la derecha.

Esta impredecibilidad de los sistemas cuánticos no implica, sin embargo, anarquía. La mecánica cuántica todavía permite que se especifiquen rigurosamente las probabilidades relativas de las distintas alternativas. Así que la mecánica cuántica es una teoría estadística. Permite predecir con precisión la evolución de conjuntos de sistemas idénticos, pero generalmente no nos dice nada preciso acerca de un sistema particular. En lo que difiere de otras teorías estadísticas, tales como la mecánica estadística, la teoría de la predicción del tiempo o la economía, es en que el elemento del azar es inherente a la naturaleza del sistema cuántico y no algo meramente 
impuesto por nuestra limitada apreciación de todas las variables que afectan el sistema.

Y no es pedante palabrería. A Einstein, por ejemplo, la idea de que hubiese una impredecibilidad inherente al mundo físico le horrorizaba tanto que la rechazó de plano, con la famosa réplica "Dios no juega a los dados con el universo". Sostuvo que la mecánica cuántica, aunque posiblemente correcta en sí misma, no deja de ser incompleta; ha de existir un nivel más profundo de variables dinámicas ocultas que afectan el sistema y sencillamente le confieren un aparente indeterminismo e impredecibilidad. Así pues, Einstein albergaba la esperanza de que bajo el caos del cuanto pudiera hallarse escondida una versión en miniatura del familiar mundo de la dinámica determinista, en la que los sobresaltos no tienen cabida.

Heisenberg y Niels Bohr se opusieron fervientemente al intento de Einstein de aferrarse a esta visión clásica del mundo. El debate, que comenzó a principios de los años treinta, se extendió durante muchos años, en los que Einstein fue perfeccionando y reformulando constantemente sus objeciones. La más pertinaz de todas ellas fue propuesta a raíz de un episodio con Boris Podolsky y Nathan Rosen en 1935, y a ella suele referirse como la paradoja de EPR (paradoja EinsteinPodolsky-Rosen) -aunque en realidad no hay tal paradoja-. Está relacionada con las propiedades de un sistema de dos partículas que interactúan y, a continuación, vuelan a gran distancia una de otra. De acuerdo con la mecánica cuántica, el sistema no deja de ser un todo indivisible, a pesar de que las partículas se separen en el espacio. Se predice que las medidas practicadas simultáneamente en las dos partículas muestran correlaciones que implican que cada partícula lleva, en algún sentido que se puede definir bien matemáticamente, una huella de la actividad de la otra partícula. Esta cooperación se produce a pesar de las restricciones de la propia teoría especial de la relatividad de Einstein, que impide cualquier comunicación física instantánea entre las partículas. 
Para Einstein, el sistema de las dos partículas demostraba que la mecánica cuántica no era completa, porque practicando las medidas sólo en la segunda partícula (utilizándola convenientemente como sustituta de la primera como medio de obtener información sobre ella) el experimentador puede deducir, bien la posición o el momento de la primera partícula en ese instante, según su capricho. Pero eso indudablemente implica, argüía Einstein, que en dicho instante ha de atribuirse un elemento de realidad a ambas cantidades, puesto que a cualquiera de ellas (jpero no a ambas!) puede acceder el experimentador $\mathrm{y}$, valiéndose de una medida que no es posible (por la restricción de la velocidad de la luz), pueda ejercer perturbación alguna sobre la partícula de interés.

La paradoja de EPR hunde sus raíces en el corazón mismo de las distintas visiones del mundo que nos vienen impuestas por la física clásica y cuántica. La visión del mundo clásica, que tan apasionadamente hace suya Einstein, casa bien con el sentido común al afirmar la realidad objetiva del mundo exterior. Reconoce la intromisión y la perturbación que nuestras observaciones inevitablemente traen consigo en dicho mundo, pero que esta perturbación es meramente incidental y puede hacerse arbitrariamente pequeña. En concreto, se considera que el micromundo de los átomos y las partículas difiere en escala, pero no en estatus ontológico, del macromundo de la experiencia. Así, un electrón es una versión en miniatura de una idealizada bola de billar, y comparte con ésta un conjunto completo de atributos dinámicos, tales como estar en algún lugar (tener una posición), moverse de un cierto modo (tener momento), etc. En un mundo clásico nuestras observaciones no crean la realidad, la descubren. De modo que átomos y partículas siguen existiendo con atributos bien definidos incluso cuando no los observamos.

Por el contrario, la interpretación de Copenague de la mecánica cuántica, que Heisenberg expone aquí tan lúcidamente, rechaza la realidad objetiva del micromundo cuántico. Niega que, pongamos por caso, un electrón tenga una 
posición bien definida y un momento bien definido, en ausencia de una observación efectiva de, bien su posición, bien su momento (y ambos no pueden dar valores precisos simultáneamente). Así que no se puede considerar un electrón o un átomo como una cosa pequeña en el mismo sentido en que se considera que una bola de billar es una cosa. Uno no puede hablar significativamente sobre lo que está haciendo un electrón entre dos observaciones sucesivas, porque son sólo las observaciones las que crean la realidad del electrón. De modo que al medir la posición de un electrón se crea un electrón con una posición; al medir su momento, se crea un electrón con un momento. Pero de ninguno de estos entes puede decirse que ya exista antes de efectuar la medida.

¿Qué es, entonces, según este punto de vista, un electrón? No es tanto una cosa física como una codificación abstracta de un conjunto de potencialidades o posibles resultados de medidas. Es un modo abreviado de referirse a un medio de poner en conexión diferentes observaciones vía el formalismo mecánico cuántico. Pero la realidad está en las observaciones, no en el electrón.

La negación de la realidad objetiva del mundo externo que la interpretación de Copenague implica suele expresarse en términos menos crudos, pero Heisenberg nos ofrece en este libro algunas de las afirmaciones más tajantes que yo haya visto jamás de esta posición. Así: «En los experimentos sobre sucesos atómicos nos las tenemos que ver con cosas y con hechos, con fenómenos que son tan reales como cualquier fenómeno de la vida diaria. Mas los átomos o las partículas elementales en sí mismos no son tan reales; forman un mundo de potencialidades o posibilidades más que un mundo de cosas o de hechos.» Las opiniones de Einstein reciben la denominación de "realismo dogmático", postura, según Heisenberg, muy natural. Es más, la inmensa mayoría de los científicos lo suscribe. Creen que sus investigaciones se refieren, de hecho, a algo real que está "ahí fuera" en el mundo físico, y que el universo físico repleto de leyes no es sólo una invención de los científicos. El éxito inesperado de 
sencillas leyes matemáticas redunda en la creencia de que la ciencia se halle llamando a la puerta de una realidad externa que ya existe. Pero, nos recuerda Heisenberg, también la mecánica cuántica tiene su fundamento en sencillas leyes matemáticas que aciertan a explicar muy satisfactoriamente el mundo físico, pero en cambio no precisan que ese mundo exista de forma independiente en el sentido del realismo dogmático. De modo que la ciencia natural es efectivamente posible sin la base del realismo dogmático.

$\mathrm{Y}$ aquí llegamos al tema que constituye la culminación de la tesis de Heisenberg. ¿Cómo podemos hablar -se pregunta- de átomos y similares si su existencia es tan oscura? ¿Qué significado hemos de atribuir a las palabras que se refieren a sus cualidades? Una y otra vez insiste en que los hechos sobre los que edificamos el mundo de la experiencia se refieren todos ellos a cosas macroscópicas -los clics de un contador Geiger, los gránulos de una placa fotográfica, etcétera. Todas éstas son cosas que podemos comunicar con pleno sentido a los demás en lenguaje liso y llano (según la frase de Bohr). Sin este telón de fondo previo de "cosas" clásicas, de sentido común, familiares (cuya realidad parece asegurada), no podemos encontrar sentido en absoluto al micromundo cuántico. Y ello debido a que todas nuestras medidas y observaciones del micromundo están referidas a un aparato clásico y suponen considerar registros bien definidos, tales como la posición de una aguja en un medidor, sobre los que todo el mundo pueda estar de acuerdo y en relación a los cuales no se suscite vaguedad o ambigüedad conceptual alguna.

Heisenberg sustenta en este punto su argumento apelando al llamado principio de complementariedad de Bohr. Este principio reconoce la esencial ambigüedad inherente a los sistemas cuánticos, el que un mismo sistema pueda poner de manifiesto propiedades aparentemente contradictorias. Por ejemplo, un electrón se puede comportar como una onda y también como una partícula. Bohr afirma que se trata de caras complementarias, y no contradictorias, de una misma realidad. Un 
experimento puede revelar la naturaleza ondulatoria del electrón y otro su naturaleza de partícula. Ambas no se pueden manifestar a la vez; corresponde al experimentador decidir qué faceta exponer al elegir su experimento. Análogamente, posición y momento son cualidades complementarias. De nuevo, ha de elegir el experimentador qué cualidad quiere observar.

La pregunta "¿es un electrón una onda o una partícula?" tiene el mismo estatus que la pregunta de si está Australia encima o debajo de Gran Bretaña. La respuesta es "ni lo uno ni lo otro y ambas cosas a la vez". El electrón posee tanto aspectos de onda como de partícula, cualquiera de los dos aspectos se puede manifestar, pero ninguno de ellos tiene significado alguno en ausencia de un contexto experimental específico. Y así, el lenguaje de la mecánica cuántica emplea palabras familiares, tales como onda, partícula, posición, etcétera, pero sus significados están severamente circunscritos y son, con frecuencia, vagos. Heisenberg nos advierte que: "Cuando este uso vago y asistemático del lenguaje nos mete en dificultades, el físico ha de retirarse a su plan matemático y su correlación sin ambigüedades con los hechos experimentales."

Éste es realmente el resultado final del argumento, puesto que la mecánica cuántica es, en esencia, un plan matemático que relaciona los resultados de las observaciones de un modo estadístico. Y eso es todo. Cualquier cosa que se diga sobre lo que "realmente" está sucediendo no es más que un intento de imbuir el mundo cuántico de una concreción espuria para que pueda imaginarse sin excesiva dificultad. A este respecto, Heisenberg examina el trabajo de Descartes y de Kant a la luz de la Física moderna y concluye que las palabras y sus conceptos asociados no tienen significados absolutos y nítidamente definidos. Surgen a raíz de nuestras experiencias en el mundo, y no sabemos de antemano los límites de su aplicación. No cabe esperar que podamos descubrir ninguna verdad fundamental sobre el mundo a partir de la mera manipulación abstracta de palabras y conceptos. Para Heisenberg, el hecho de que ciertas palabras y conceptos que nos son muy queridos sencillamente 
no se puedan transportar a los dominios de la relatividad o de lo cuántico no es especialmente objetable filosóficamente.

Aunque la mayor parte del debate cuántico se ha desarrollado a un nivel filosófico, ha habido un número de experimentos cruciales directamente relacionados con el tema. Quizá el más importante es el que se refiere a la elevación del experimento imaginado por EPR al ámbito de la física práctica. En 1965 John Bell llevó más lejos el argumento de EPR y demostró que, dicho sin excesivo rigor, cualquier teoría basada en la "realidad objetiva", y en la cual no se admita que ninguna señal viaje más deprisa que la luz, ha de satisfacer ciertas desigualdades matemáticas. De acuerdo con la teoría estándar, la mecánica cuántica no debería poder satisfacer tales desigualdades, de manera que uno se ve en la obligación de renunciar a la realidad objetiva (con Bohr y Heisenberg) o a la teoría de la relatividad. Pocos físicos están dispuestos a tomar este último camino. Para contrastar las desigualdades de Bell, a principios de los años ochenta Alain Aspect y sus colegas del Institut d'Optique, cerca de París, realizaron experimentos utilizando pares de fotones procedentes de una fuente atómica común. Tras muchas y cuidadosas pruebas los resultados estaban claros: se violaban notoriamente las desigualdades de Bell, tal y como predecía la mecánica cuántica.

Estos resultados llegaron después de la muerte de Heisenberg, pero yo tuve ocasión de discutirlos con muchos de sus antiguos colegas quienes, junto con Bohr, habían contribuido a dar forma a la interpretación de Copenague en los años treinta. Ninguno de ellos se mostraba excesivamente entusiasta acerca del experimento de Aspect, que tan bellamente reforzaba su posición, manifestando que los resultados no podrían haber sido otros y que no eran ninguna sorpresa.

No obstante lo anterior, a la interpretación de Copenague no le faltan sus detractores. Muchos físicos no acaban de sentirse cómodos con una teoría en la que el formalismo 
ha de aumentarse con ciertos supuestos epistemológicos antes de poderse aplicar. El hecho de que la interpretación de Copenague se base en la aceptación de la existencia previa del mundo macroscópico clásico parece circular y paradójico, puesto que el macromundo se compone del micromundo cuántico. Aunque los efectos cuánticos en las agujas de los medidores y en los gránulos fotográficos son casi despreciables, en principio están ahí. A los físicos les gustaría derivar el mundo clásico como algún tipo de límite macroscópico del mundo cuántico, no suponerlo a priori.

El punto débil de la interpretación de Copenague se presenta cuando se plantea la siguiente cuestión: ¿Qué sucede realmente dentro de un pedazo del aparato medidor cuando se realiza una medida de una partícula cuántica? La posición de Copenague es que uno sencillamente trata el aparato clásicamente; pero si (con más realismo) se trata por el contrario como un conjunto (aunque grande) de partículas cuánticas, entonces el resultado es ciertamente preocupante. La misma vaguedad e indeterminismo que afecta a la partícula cuántica invade ahora todo el sistema. En vez de que el aparato concrete una realidad específica, de entre un abanico de posibilidades potenciales, el sistema combinado de aparato+partícula adopta un estado que sigue representando un abanico de posibilidades potenciales. Por poner un ejemplo concreto, si el aparato se coloca para que mida si un electrón está en la mitad derecha o izquierda de una caja, y para indicarlo debe llevar la aguja a la derecha o a la izquierda, respectivamente, el resultado final del ejercicio es que el sistema combinado se sitúa en un estado en el que no se selecciona ni uno ni otro resultado, sino que el estado es una superposición de dos estados, uno que consiste en que el electrón y la aguja estén a la derecha, el otro en que estén a la izquierda. En tanto estas dos alternativas sean mutuamente excluyentes, podría no tratarse de un problema insuperable, pero en experimentos más generales puede también haber interferencias entre las alternativas, de modo que no se presente una dicotomía clara entre ellas. En resumen, no se puede decir que se haya producido medida efectiva alguna. 
Heisenberg dedica escasa atención al voluminoso trabajo sobre el "problema de la medida" de John von Neumann y otros autores. Retrocede acogiéndose al argumento de que, antes o después, los efectos cuánticos (en concreto la interferencia de las posibilidades) acaban por disiparse en el medio macroscópico. Lo cual satisfará a casi todo el mundo, mas no a una moderna raza de físicos conocida como cosmológica cuántica. Estos teóricos intentan aplicar la mecánica cuántica al universo en su conjunto en un esfuerzo por desentrañar el misterio de su origen. Si el sistema cuántico de interés es todo el universo, resulta evidente que ya no hay un medio, o aparato de medida macroscópico más amplio capaz de despejar la bruma cuántica. La mayoría de los cosmólogos cuánticos rechazan la interpretación de Copenague, con su necesidad de maquinaria epistemológica adicional, y prefieren, en cambio, tomar el formalismo cuántico tal y como se presenta literalmente at face value. Lo cual significa aceptar serenamente el abanico completo de alternativas cuánticas como realidades efectivamente existentes. Esto es, en el experimento de medición arriba mencionado, uno daría por cierta la existencia de dos universos, uno con el electrón y la aguja a la izquierda y el otro con ellos a la derecha. En general, una medida cuántica supone postular una infinidad de mundos, o realidades, paralelos coexistentes. De nuevo, muchos de estos desarrollos se han realizado tras la muerte de Heisengberg, si bien sospecho que él no les habría dedicado excesiva atención.

Este libro también toca otros temas, entre los que destacan muy especialmente algunos de los primeros avances en la Física nuclear y de partículas. Heisenberg no se detiene mucho en sus propios intentos por unificar la física de partículas, pero sí señala algunas dificultades muy serias que surgen al aplicar la mecánica cuántica a las partículas relativistas. Aquí, también, los acontecimientos han superado el libro. Las temidas divergencias, o infinitudes que él menciona, se acomodan hoy, de forma rutinaria, en casi todas las aplicaciones, sin arruinar la capacidad de predicción de la teoría. Es más, en ciertas teorías unificadas modernas, especialmente en la llamada teoría de las supercuerdas, es posible evitarlas del todo. También nuestra teoría de 
las partículas elementales se halla hoy en una incomparablemente mejor forma que cuando el libro fue escrito, y la teoría moderna de los quarks y los leptones habría contado con la aprobación de Heisenberg. Su discusión de Dios y la moral es más bien superficial, y es mi sospecha que su inclusión se debe en gran medida a la necesidad de satisfacer las exigencias de las clases magistrales Gifford.

Pero son estas objeciones menores a un libro que tan satisfactoriamente disecciona la esencia de la revolución conceptual que la Nueva Física supone. Heisenberg logra este objetivo sin hacer uso de las matemáticas y reduciendo al mínimo los detalles técnicos. Ciertamente uno no tiene que ser físico para seguir sus argumentos y para apreciar la naturaleza momentánea del cambio de paradigma que siguió a las revoluciones relativista y cuántica. El atractivo de este libro, que tan bien resiste el paso del tiempo, está en que lleva al lector, con una claridad digna de destacar, del esotérico mundo de la Física atómica al mundo de las personas, el lenguaje y la concepción de la realidad que compartimos. 